\title{
Impact of cell load on 5GHz IEEE 802.11 WLAN
}

\author{
Mamun Abu-Tair*, Saleem N. Bhatti** \\ ${ }^{*}$ School of Computing and Information Engineering, Ulster University, UK \\ ${ }^{* *}$ School of Computer Science, University of St Andrews, UK \\ *m.abu-tair@ulster.ac.uk, ${ }^{* *}$ saleem@st-andrews.ac.uk
}

\begin{abstract}
We have conducted an empirical study of the latest 5GHz IEEE 802.11 wireless LAN (WLAN) variants of 802.11n (5GHz) and 802.11ac (Wave 1), under different cell load conditions. We have considered typical configurations of both protocols on a Linux testbed. Under light load, there is no clear difference between 802.11n and 802.11ac in terms of performance and energy consumption. However, in some cases of high cell load, we have found that there may be a small advantage with 802.11ac. Overall, we conclude that there may be little benefit in upgrading from $802.11 n(5 \mathrm{GHz})$ to $802.11 \mathrm{ac}$ in its current offering, as the benefits may be too small.
\end{abstract}

\section{INTRODUCTION}

The Institute of Electrical and Electronics Engineers (IEEE) have ratified a new variant of 802.11 family, $802.11 \mathrm{ac}$, which aims to improve the network throughput over its predecessor $802.11 \mathrm{n}$. To ensure maximum network throughput, 802.11ac has the capability to use wider channels, with 80 and 160 $\mathrm{MHz}$ channel width, compared to $20 \mathrm{MHz}$ and $40 \mathrm{MHz}$ in $802.11 \mathrm{n}$. However, the greater channel widths might not be usable especially in crowded WLAN environments.

One of the main factors that can affect the performance of WLANs is load for a given WLAN cell (Access Point) - also referred to as the Basic Service Set (BSS). While there are common procedures and modules across different variants of 802.11, there are also differences in handling the shared media to provide more network throughput for the end user. The most recent 802.11 protocols have the ability to use High Throughput (HT - 802.11n and 802.11ac) and Very High Throughput (VHT - 80.11ac) channels, to handle more data within the BSS.

When considering upgrades from $802.11 \mathrm{n}$ to $802.11 \mathrm{ac}$, the cost of such upgrades must be balanced against potential advantages and actual benefits from real use. 802.11n WLAN can support $20 \mathrm{MHz}$ and $40 \mathrm{MHz}$ channels, while $802.11 \mathrm{ac}$ can support $20 \mathrm{MHz}, 40 \mathrm{MHz}, 80 \mathrm{MHz}$ and $160 \mathrm{MHz}$. The wider channels are desirable for increased throughput. However, with current deployment scenarios in office and campus environments, based on managed cell-plans, it is likely that the $20 \mathrm{MHz}$ and $40 \mathrm{MHz}$ width channels remain in use to avoid radio interference between neighbouring cells.

Therefore, especially in existing planned network topologies (e.g. a campus or an office), 802.11ac may still have to use $20 \mathrm{MHz}$ and $40 \mathrm{MHz}$ channels. In such conditions, it is not obvious that there will be performance gains when moving from $802.11 \mathrm{n}$ to $802.11 \mathrm{ac}$, and it is instructive to compare the performance of $802.11 \mathrm{ac}$ with $802.11 \mathrm{n}$ under such conditions.

\section{A. Motivation and Approach}

We have conducted an empirical study to compare the performance of 802.11n and 802.11ac under different levels of cell load. We have used the current Wave 1 of $802.11 \mathrm{ac}$, which does not include multi-user multiple-input multiple output (MU-MIMO) functions. The testbed that is used in this study is configured with a common deployment configuration. We test throughput with the Transmission Control Protocol (TCP), which is the most widely used transport protocol on the Internet. TCP supports different types of application such as email, WWW access and video streaming. In keeping with the methodology of our previous work [1]-[4], our approach is empirical, based on measurements of performance and energy usage of real systems.

We use off-the-shelf equipment, opensource software, and consumer devices wherever possible. Our intentions are:

- To examine systems that are typical of normal usage, so that our results reflect real operational scenarios, rather than lab-specific, optimised configurations.

- Make it possible to apply our methodology easily to other similar scenarios, to allow comparisons.

- Allow our results to be validated / reproduced easily.

\section{B. Contribution and structure of this paper}

Our main finding is that moving from $802.11 \mathrm{n}$ to $802.11 \mathrm{ac}$ may not be beneficial if $20 \mathrm{MHz}$ and $40 \mathrm{MHz}$ channels are used, i.e. for existing network deployments, there may be little benefit in replacing $802.11 \mathrm{n}$ base-stations with 802.11 ac basestations in an attempt to improve performance. At the very least, new network cell-plans may be required, which may also require re-wiring and re-siting of base-stations for optimum performance with 802.11ac.

We have conducted an empirical study in the performance of WLANs and considered client-side performance. We have examined the throughput and energy usage of the $802.11 \mathrm{n}$ and 802.11ac at $5 \mathrm{GHz}$ using $20 \mathrm{MHz}$ and $40 \mathrm{MHz}$ channel widths. All the experiments have been conducted under different cell loads to provide a complete picture of WLAN performance from a client view-point. We evaluated performance using TCP flows, and we also examined energy usage of the two 802.11 variants. We considered CUBIC as the TCP congestion control protocol [5] (the default for Linux).

In Section II we provide information on background and related work. Section III explains our methodology and describes our testbed. We discuss our results in Section IV, and conclude with a short summary in Section V. 


\section{RELATED WORK AND BACKGROUND}

\section{A. RF channel options in WLANs}

WLANs operate in two Industry, Scientific, Medical (ISM) bands, $2.4 \mathrm{GHz}$ and $5 \mathrm{GHz}$. The Radio Frequency (RF) transmission characteristics of 802.11 radio are described in the IEEE 802.11 standard, which includes the channelisation scheme as well as the spectrum radiation of the signal [6].

In this study we consider $5 \mathrm{GHz}$ WLANs only, for the following reasons:

- the $2.4 \mathrm{GHz}$ band is generally considered to be saturated and will yield no further benefit which is cost effective.

- the $5 \mathrm{GHz}$ band has non-overlapping channels.

- the $5 \mathrm{GHz}$ band has more channels compared to the $2.4 \mathrm{GHz}$ band.

- $5 \mathrm{GHz}$ WLAN is becoming widely deployed, and mature networks and client deployments exist.

- the latest $802.11 \mathrm{ac}$ protocol operates on $5 \mathrm{GHz}$ band only.

The $5 \mathrm{GHz}$ band (see Table I) has several sub-bands. UNII-1 is widely available, globally, as is UNII-3, but the latter may not be supported so widely in equipment, especially cheaper or low-end client devices. The UNII-2 and UNII-2-Ext subbands require the use of Dynamic Frequency Selection (DFS) algorithms for avoiding radar systems (e.g. weather radar). So, UNII-2 and UNII-2-Ext sub-bands might not be so widely available for use, as well as not being implemented in some equipment. Hence, for many systems, it may be that only UNII-1 is available for use. So, using a $20 \mathrm{MHz}$ channel from the UNII-1 band will allow four non-overlapping APs to operate in the same area. In case of a $40 \mathrm{MHz}$ channel, only two non-overlapping APs can operate in the same area.

TABLE I

5GHz WLAN BANDS, EACH CHANNEL IS 20MHz

\begin{tabular}{|l|l|}
\hline 5 GHz sub-band & Channel numbers \\
\hline UNII-1 & $36,40,44,48$ \\
\hline UNII-2 & $52,56,60,64$ \\
\hline UNII-2-Ext & $100,104,108,112,116,120$ \\
& $124,128,132,136,140$ \\
\hline UNII-3 & $149,153,157,161,165$ \\
\hline
\end{tabular}

UNII - Unlicensed National Information Infrastructure

Of course, if UNII-3 is available, then it is possible to have more non-overlapping APs. Deek et al have previously examined the use of a $40 \mathrm{MHz}$ channel, and find some constraints on its use also [7]. Moving to an $80 \mathrm{MHz}$ channel would allow only a 2-cell plan, and using a 160MHZ channel (e.g. as $80 \mathrm{MHz}$ UNII-1 + 80MHz UNII-3) would only allow a single cell.

For our experiments, we have taken the position that 20 $\mathrm{MHz}$ and $40 \mathrm{MHz}$ channels are likely to remain in use for some time with 5GHz WLANs, for 802.11n and 802.11ac [8].

\section{B. TCP behaviour}

The Transmission Control (TCP) protocol is the most widely used protocol on the Internet. TCP is used for email, file transfer, web access, video streaming and many other applications. The main aim of TCP protocols is to provide endto-end reliable data transmission with a congestion control mechanism in order to adjust the transmission rate, avoiding end-to-end loss. TCPs original congestion control behaviour was defined by Jacobson in 1988 [9].

In this study, we consider a widely used TCP variant called CUBIC [5]. CUBIC is the Linux default TCP variant, and is designed for better performance on paths that have high throughput and/or paths with a long round-trip-time (RTT).

\section{WLAN performance and energy usage}

This paper takes another aspect of the performance and energy analysis of 802.11 WLANs, as a complement to our previous work in this area [1]-[3], [8], [10], [11]. Our previous work [8] presented our initial empirical results of 802.11ac and $802.11 \mathrm{n}$ performance in an office scenario. This study considered the following new results to draw a full picture of 802.11ac and 802.11n comparison:

- a comparison of both $20 \mathrm{MHz}$ and $40 \mathrm{MHz}$ channels for both $802.11 \mathrm{n}$ and $802.11 \mathrm{ac}$.

- consideration of competition for media access, by the use of background traffic with various cell loads (upto 75\%), at various background traffic rates (upto $165 \mathrm{Mbps}$ ).

- examination of energy usage of TCP for the client systems as a whole, for 802.11n and 802.11ac.

Our previous work [1] established the use of the energy metric, $E_{A}$, and the notion of the energy envelope, which gives the upper and lower bounds of the energy usage during the transmission of a flow. We have also investigated the possibility of application adaptation within the scope of this energy envelope [2] to trade off performance against energy usage. Also, we have found that the generic 802.11 power save mode (PSM) has little effect during system usage [10]. In [11] we addressed the problem of the impact on WLAN performance due to low Received Signal Strength Indication (RSSI) in WLAN cells. Those studies all includes measurements in both the $2.4 \mathrm{GHz}$ and $5 \mathrm{GHz}$ bands. We have also examined the energy usage of the Datagram Congestion Control protocol (DCCP) and User Datagram Protocol (UDP) at different packet sizes and data rates over 802.11n WLAN at $5 \mathrm{GHz}$ [3]. The results show DCCP can provide $\sim 10 \%$ to $\sim 40 \%$ greater energy efficiency than the UDP. Also, we have considered how newer $5 \mathrm{GHz} 802.11$ systems perform when legacy $5 \mathrm{GHz}$ clients are in use [4].

Zeng et al [12] have evaluated 802.11ac performance. They observed that throughput, and so energy usage, was very variable, but that 802.11 ac can achieve higher throughput overall when using wide RF channels $(40 \mathrm{MHz}$ and greater). The authors provided a comparison between 802.11n and 802.11ac protocols, but the $802.11 \mathrm{n}$ experiments used $40 \mathrm{MHz}$ channels while the $802.11 \mathrm{ac}$ experiments used $80 \mathrm{MHz}$ channels.

Keranidis et al [13] have considered an experimental comparison of energy efficiency for 802.11n. However, they evaluated the energy consumption for the Network Interface Card (NIC) only, while in this paper and our previous work, we 
consider the impact on the client system as a whole, as that will be the real impact observed by users. Additionally, their study used optimisations to system configuration to improve energy efficiency, whilst we take the position that users normally adopt default configurations.

Halpern et al [14] provided an empirical study of the power consumption of 802.11n WLAN but again only considered the NIC. Their study concluded that the use of larger packets and higher date rate in transmission is more energy efficient than using a smaller packet size and lower data rates.

$\mathrm{Li}$ et al [15] also examined the impact of packet size on the energy consumption in heterogeneous wireless network environments, with similar observations: larger packet sizes and higher data rates give better energy efficiency.

\section{EXPERIMENT DESIGN AND METRICS}

We measured performance and energy usage of TCP flows transmitted over $802.11 \mathrm{n}$ and $802.11 \mathrm{ac}$ at $5 \mathrm{GHz}$, in a modern, open-plan office environment. We used opensource software, off-the-shelf hardware, and default configurations for all systems, unless otherwise detailed below.

\section{A. Overview}

The testbed (Figure 1) consisted of two clients systems - a UDP client generated background traffic (to generate load in the cell) and the TCP client generated a TCP flow for which we measured performance. An additional host acted as both a wireless access-point (AP) and as a server (the other endpoint of the traffic flows). All hosts were set up in a teaching lab in the University of St Andrews. The distance between the server and the TCP client was $\sim 10 \mathrm{~m}$ and the other client was placed at half the distance between them. iperf v2.0.2 ${ }^{1}$ was used in server mode to receive traffic flows. The energy usage of the TCP client was measured by using a commercial power meter. CUBIC TCP was used at the server and at the TCP client. Each TCP iperf measurement was a $100 \mathrm{MB}$ transfer $(800 \mathrm{Mb}$, enough to exercise the TCP algorithms), and was performed 30 times for each of the two network configurations $(20 \mathrm{MHz}$ and $40 \mathrm{MHz}$ channels) against 8 different background loads and 4 different cell loads, giving 720 measurements in total.

\section{B. Equipment}

Our testbed was equipped with identical hosts. The hardware specifications of the clients and the server were: a Scan home-office PC $\left(\mathrm{VIO}^{2}\right)$ with an Intel $\AA$ Core i5 $44403.1 \mathrm{GHz}$ Quad Core CPU, 4GB RAM, 1TB HD. All machines used the same wireless LAN NIC hardware ${ }^{3}$ based on the QCA9880 Version 2 Atheros ${ }^{4}$ chipset, with $3 \times 3$ MIMO. Our power meter was an i-Sockets ${ }^{5}$ instrumented, domestic, multi-way power extension.

\footnotetext{
${ }^{1}$ http://www.erg.abdn.ac.uk/users/gerrit/dccp/apps/\#iperf

${ }^{2}$ http://3xs.scan.co.uk/configurator/ready-to-ship-budget-value-amd-homeoffice-pc-v10a

${ }^{3}$ http://www.compex.com.sg/Datasheets/WLE900VX_Dsv1.0.1-140711I.pdf

${ }^{4}$ http://www.atheros.com/

${ }^{5}$ http://www.i-sockets.com/
}

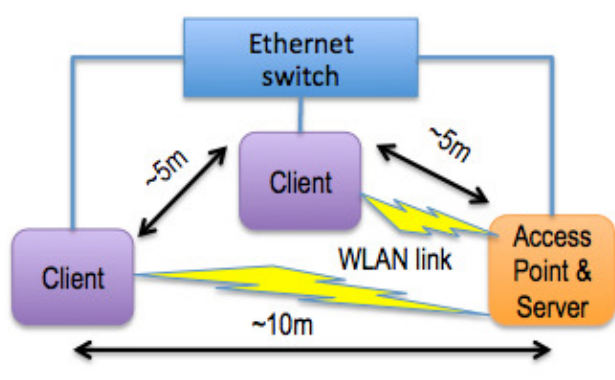

Fig. 1. Schematic of testbed showing physical connectivity. All experiments used $802.11 \mathrm{n}$ and $802.11 \mathrm{ac}$ at $5 \mathrm{GHz}$ with $20 \mathrm{MHz}$ and $40 \mathrm{MHz}$ channels. The experiments used Ethernet for a control channel and file-system access. Only test-traffic traversed the WLAN link. The antennas of the TCP client and access point / server were $\sim 10 \pm 0.5 \mathrm{~m}$ apart. A client that generated background traffic was placed at $\sim 5 \mathrm{~m}$ from the AP. The TCP flows generated using iperf v2.0.2 were transferred across the WLAN link.

Ubuntu 14.04 was used on each host, a minimal server distribution, with the Linux kernel version 3.16.0-wl-ath+, using the latest ath10k driver ${ }^{6}$. For implementing the AP, we used the hostapd ${ }^{7}$ package version v2.3-devel. All nodes in the testbed were connected via a local ethernet network which was also used for controlling the experiment: only test traffic traversed the WLAN link. The Linux utility iwconfig ${ }^{8}$ was used to record the link quality and the signal level of the RF channel.

\section{Performance Metrics}

From iperf we recorded for each TCP flow the end-to-end datarate, $r$. The energy metric used, $E_{A}$, had units microJoules per bit $(\mu J / b)$ (Eqn. 1), which is numerically equal to Joules per megabit. For each flow, we measured the mean power usage over the duration of the flow $\left(P_{F}\right)$, and subtracted the idle power $\left(P_{I}\right)$ measured for the client $(36 \mathrm{~W})$, then divided by the throughput $(r)$. Lower values of $E_{A}$ are better.

$$
E_{A}=\frac{P_{F}-P_{I}}{r}
$$

TABLE II

MAIN METRICS FOR EVALUATION

\begin{tabular}{|c|l|l|l|}
\hline Metric & Description & Units & Comment \\
\hline$E_{A}$ & energy usage of flow & $\mu \mathrm{J} / \mathrm{b}$ & Eqn. 1 \\
\hline$r$ & TCP throughput & Mbps & from iperf \\
\hline
\end{tabular}

Additionally, link quality and signal strength of the WLAN RF channel was monitored during the experiments, as reported by the WLAN NIC driver. This was used to confirm that there were no anomalous or exceptional fluctuations in the RF transmission conditions during the tests.

\section{RESUlts AND Discussion}

In this section, we provide an analysis and discussion of the results obtained from our experiments. Firstly, in Section

\footnotetext{
${ }^{6} \mathrm{https}: / /$ github.com/kvalo/ath10k

${ }^{7}$ http://hostap.epitest.fi/hostapd/

${ }^{8} \mathrm{http}: / /$ www.linuxcommand.org/man_pages/iwconfig8.html
} 
IV-A, we provide a comparison of performance and energy usage between $802.11 \mathrm{ac}$ and $802.11 \mathrm{n}$ in a best-case WLAN scenario where there is no competition for the shared channel. Section IV-B discusses the result of WLAN performance and energy usage for a single TCP client, under different cell loads (the latter generated by the UDP client). Section IV-C, suggests an adjustment that can be made to the WLAN that can improve performance for the end user. Section IV-D discusses the context of the study. Finally Section IV-E discusses 802.11ac performance and the forthcoming features for Wave 2 of $802.11 \mathrm{ac}$.

\section{A. Best Case WLAN Scenario}

Providing extra throughput for WLANs was the main motivation of almost all ratified 802.11 variants, including the latest 802.11n and 802.11ac variants. However, 802.11 protocols interact in different ways according to the operating environment. In Figures 2 and 3 , point 0 on the $\mathrm{x}$-axis indicates that there is no background traffic and no other client competing for the shared channel. In terms of throughput, it is clear from the figure that there is little difference in performance between $802.11 \mathrm{n}$ and $802.11 \mathrm{ac}$ when using a $20 \mathrm{MHz}$ channel width. However, for a $40 \mathrm{MHz}$ channel width, 802.11 n has $\sim 30 \%$ and $\sim 12 \%$ better throughput compared to 802.11 ac. In terms of energy usage, for both channel widths, $802.11 \mathrm{n}$ and 802.11ac have almost the same energy usage. However, by comparing Figure $2 \mathrm{c}$ with Figure $3 \mathrm{c}$, the benefit of using a $40 \mathrm{MHz}$ channel width over the $20 \mathrm{MHz}$ channel width is very clear in terms of energy usage. This is simply due to the fact that the $40 \mathrm{MHz}$ channel is delivering more data compared to the $20 \mathrm{MHz}$ channel network over the same time, amortising the energy usage of the hosts over more bits.

Key observation: $802.11 \mathrm{n}$ performs similarly to, or better than, 802.11ac when there is no competition for the wireless channel.

\section{B. WLAN cells under load}

For scenarios such as the Internet of Things (IoT), almost every device in future smart homes will have its own wireless interface. This will increase the competition for the shared wireless channel between those devices. Therefore, investigating the performance of the WLAN cell under different cell loads is an important aspect to find how different protocols act under different load scenarios. Figure 2 a shows the throughput of the TCP flows under different background traffic loads for $802.11 \mathrm{n}$ and $802.11 \mathrm{ac}$ operating on a $20 \mathrm{MHz}$ channel. As shown in the figure, under zero and light background load $(\leq 25 \mathrm{Mbps})$ there is no clear difference between the two 802.11 variants. When the background traffic load increases beyond 50Mbps, 802.11ac does perform better than $802.11 \mathrm{n}$. Figure $2 \mathrm{~b}$ shows the throughput of $802.11 \mathrm{ac}$ and $802.11 \mathrm{n}$ under different cell loads. It is clear from the figure that $802.11 \mathrm{ac}$ has a slightly better performance compared to $8012.11 \mathrm{n}$ in extremely busy cells (when the load is above $50 \%$ ).

Key observation: Using a $20 \mathrm{MHz}$ channel, $802.11 \mathrm{ac}$ performs slightly better than 802.11 n on very busy WLAN cells
(50\% load or higher) in terms of throughput.

With a $40 \mathrm{MHz}$ channel, Figure 3a shows the throughput of 802.11ac and 802.11n under different background loads. 802.11 ac has better performance over 802.11 n only when the background traffic loads is $\geq 125 \mathrm{Mbps}$. Otherwise, $802.11 \mathrm{n}$ performed better or similar to 802.11ac. Figure 3d illustrates this finding, and shows that 802.11ac has a clear advantage over $802.11 \mathrm{n}$ above $\sim 50 \%$ cell loads.

In terms of energy usage, $802.11 \mathrm{n}$ and $802.11 \mathrm{ac}$ have almost the same energy usage with zero and lightly loaded cells and with different channel widths. However, 802.11ac has better energy efficiency than 802.11 under heavily loaded cells. For example, Figure 2c shows $E_{A}$ for TCP flows of both 802.11 protocols using a $20 \mathrm{MHz}$ channel width. From the graph, when the background load is $\leq 25 \mathrm{Mbps}$ there is no difference in energy usage between the two WLAN variants. However, when the background traffic exceeds $25 \mathrm{Mbps}$, 802.11ac has better energy efficiency compared to $802.11 \mathrm{n}$. This is due to the fact that 802.11ac delivers more data than 802.11n in the heavy loaded WLAN cells.

Key observations:

- 802.11ac saves energy on heavily loaded WLAN cell scenarios compared to $802.11 \mathrm{n}$.

- Using a $40 \mathrm{MHz}$ channel width if possible, is more energy efficient for both 802.11n and 802.11ac.

\section{Reducing upgrade costs}

It is clear from our discussion above that the current state of 802.11ac offers no significant improvement compared to its predecessor $802.11 \mathrm{n}$ especially with narrow width channels. With increasing use of WLANs, the $80 \mathrm{MHz}$ and $160 \mathrm{MHz}$ channels are unlikely to be used widely, as such wide channels may be impacted by interference from neighbouring cells. However, tuning some system parameters may increase the performance of a WLAN client without the need to upgrade to a new 802.11 variant, which would require purchase of new equipment. For example, using a WLAN management algorithm that can sense the usage of the nearby channels and switch to a $40 \mathrm{MHz}$ channel if possible to increase the performance of the WLAN clients.

\section{Our observations in context}

Our testbed measured transmission between the access point and a client directly connected via the WLAN link. We do not consider an Internet-wide end-to-end path. The applicability of our measurements is therefore on a local access scenario, where the client systems serve users with access to local systems. Relevant uses cases might be, for example:

- Domestic scenario: modern broadband routers now also allow plug-in data storage, for example, either flash memory (e.g. USB flash drives), or hard drives (again via USB, or built-in internally). These might be used to serve locally shared files or media, and the WLAN AP is also the server. 
- Small office / Home office (SoHo): local files stored on a server that is close to the AP, e.g. connected via a highspeed (Gbps) ethernet link.

- Campus: Campus environments supporting high density of WLAN APs, connected to a Gbps ethernet switch and then to high-capacity servers for various services.

Also, many of these scenarios now involve local caching, e.g. campus proxy servers for the web, home multi-play gateway/servers offer video on demand and other services. This is analogous to the AP acting as a local server in our testbed. However, if an Internet-wide end-to-end path was considered, where performance bottlenecks and/or packet drop points existed at other points along the path, and the RTT of the path as a whole was higher, we would likely see different performance from that measured in our scenario.

\section{E. 802.11ac performance: near-term futures}

While the similarity between our measurements of $802.11 \mathrm{ac}$ may seem surprising - showing little improvement compared to $802.11 \mathrm{n}$ - such results are to be expected. The current (Wave 1) 802.11ac products use MIMO and $20 \mathrm{MHz}$ or 40 $\mathrm{MHz}$ channels, as does 802.11n. 802.11ac does introduce some additional modulation coding scheme (MCS) points, however. Our testbed used $3 \times 3$ MIMO, but $2 \times 2$ is common in lowend equipment, both of which can be used by $802.11 \mathrm{ac}$ and $802.11 \mathrm{n}$. When $802.1 \mathrm{ac}$ does allow $80 \mathrm{MHz}$ and $160 \mathrm{MHz}$ channels, often only a single such channel is possible, which makes deployment impractical where neighbouring cells are in use. So, there is not much difference in terms of physical-level resources between the systems.

However, the next round of 802.11ac equipment (Wave 2, appearing for purchase at the time of writing), will feature enhancements beyond 802.11n. These include multi-user MIMO (MIMO), which will allow multiple users to send to the AP simultaneously, using beam-forming technologies. This aims to improve overall throughput for individual clients through reduced contention for a single channel. Such improvements will need upgrades to APs, but it may be possible that some client equipment can work with these APs with little modification (e.g. software and/or firmware upgrades). 802.11ac can also use upto 8 antennas for $8 \times 8$ MIMO, which will also provide improved capacity, but will require upgrades to APs and client hardware.

However, for scenarios such as the Internet of Things (IoT), if 802.11 WLAN is used, then the devices (clients) may be too small to have sufficient battery power and appropriate geometry to support multiple antennae and MU-MIMO features such as beamforming. Of course, there is much activity in defining other (new) RF systems for IoT also. However, similar arguments of battery life and limitations of size and geometry may also limit the use of 802.11ac features for handheld devices such as smartphones, tablets and wearables.

\section{CONCLUSiON}

We have conducted experiments to measure the performance and energy usage of a $802.11 \mathrm{n}$ and $802.11 \mathrm{ac}$ at $5 \mathrm{GHz}$ in a local access scenario, using the Transmission Control Protocol (TCP). In our experiments, we used the most common channel widths deployed for the WLAN Access Point (AP) today, 20 $\mathrm{MHz}$ and $40 \mathrm{MHz}$ channel widths. We find that in the current state of systems development, the performance difference between $802.11 \mathrm{n}$ to $802.11 \mathrm{ac}$ is not substantial, and so may not merit the expense of a large upgrade where a mature $802.11 \mathrm{n}$ deployment exists.

\section{ACKNOWLEDGEMENTS}

This work has been supported by the IU-ATC project (http://www.iu-atc.com/), funded by grant EP/J016756/1, awarded by the Engineering and Physical Sciences Research Council (EPSRC), UK.

\section{REFERENCES}

[1] M. Tauber, S. N. Bhatti, and Y. Yu, "Application Level Energy and Performance Measurements in a Wireless LAN," in GreenCom 2011 IEEE/ACM Intl. Conf. Green Computing and Comms., Aug 2011.

[2] — "Towards Energy-Awareness in Managing Wireless LAN Applications," in NOMS 2012 - IEEE/IFIP Network Operations and Management Symp., Apr 2012.

[3] M. Abu-Tair and S. N. Bhatti, "Energy Usage of UDP and DCCP over 802.11n," in IEEE AINA 2014 - 28th Intl. Conf. Advanced Information Networking and Applications, 2014, pp. 313-320.

[4] — , "IEEE 802.11ac MU-MIMO Wireless LAN cells with Legacy Clients," in AINA-2017 - 31st IEEE Intl. Conf. on Advanced Information Networking and Applications, Mar 2017.

[5] L. Xu and I. Rhee, "CUBIC: A new TCP-Friendly high-speed TCP variant," in PFLDNet 2005 - 3rd. Int. Wkshp. Protocols for Fast LongDistance Networks, Feb 2005.

[6] M.-J. Ho, M. Rawles, M. Vrijkorte, and L. Fei, "Rf challenges for 2.4 and 5 ghz wlan deployment and design," pp. 783-788 vol.2, Mar 2002.

[7] L. Deek, E. Garcia-Villegas, E. Belding, S.-J. Lee, and K. Almeroth, "The Impact of Channel Bonding on 802.11N Network Management," in Proc. ACM CONEXT 2011 - 7th COnference on Emerging Networking EXperiments and Technologies, 2011, pp. 11:1-11:12.

[8] M. Abu-Tair and S. N. Bhatti, "Introducing IEEE 802.11ac into existing WLAN deployment scenarios," in WiOpt2015 - 13th Intl. Symp. on Modeling and Optimization in Mobile, Ad Hoc, and Wireless Networks, May 2015.

[9] V. Jacobsen, "Congestion avoidance and control," in Proc. ACM SIGCOMM 1988, Aug 1988.

[10] M. Tauber and S. N. Bhatti, "The Effect of the 802.11 Power Save Mechanism (PSM) on Energy Efficiency and Performance During System Activity," in GreenCom 2012 - IEEE Intl. Conf. Green Computing and Communications, Nov 2012, pp. 573-580.

[11] M. Tauber and S. Bhatti, "Low RSSI in WLANs: Impact on applicationlevel performance," in ICNC2013 - 2013 Intl. Conf. on Computing, Networking and Communications, 2013, pp. 123-129.

[12] Y. Zeng, P. H. Pathak, and P. Mohapatra, "A First Look at 802.11ac in Action: Energy Efficiency and Interference Characterization," in Networking2014 - IFIP Intl. Conf. on Networking, Jun 2014.

[13] S. Keranidis, G. Kazdaridis, N. Makris, T. Korakis, I. Koutsopoulos, and L. Tassiulas, "Experimental Evaluation and Comparative Study on Energy Efficiency of the Evolving IEEE 802.11 Standards," in Proc. e-energy2014 - 5th ACM Intl. Conf. on Future Energy Systems, ser. eEnergy '14. New York, NY, USA: ACM, 2014, pp. 109-119.

[14] D. Halperin, B. Greensteiny, A. Shethy, and D. Wetherall, "Demystifying 802.11n power consumption," in 2010 Wkshp. on Power Aware Computing and Systems, ser. HotPower'10. Berkeley, CA, USA: USENIX Association, 2010.

[15] Y. Li, X. Qi, M. Keally, Z. Ren, G. Zhou, D. Xiao, and S. Deng, "Communication Energy Modeling and Optimization through Joint Packet Size Analysis of BSN and WiFi Networks," IEEE Trans. Parallel and Distributed Systems, vol. 24, no. 9, pp. 1741-1751, 2013. 


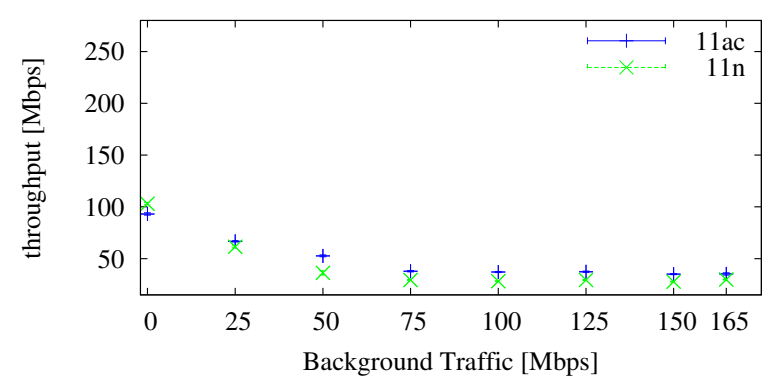

(a) Throughput

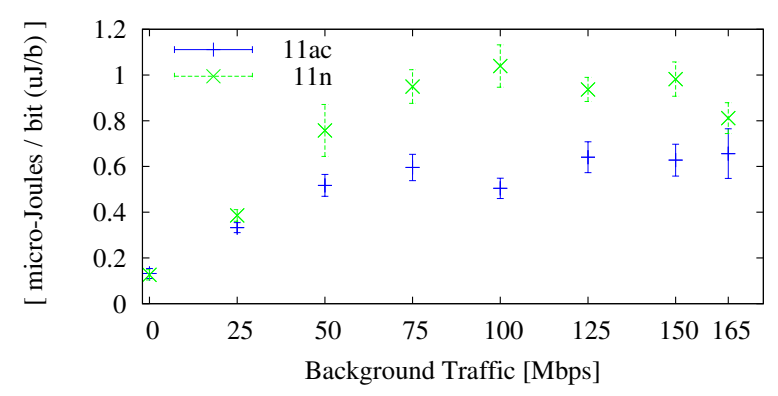

(c) Energy usage

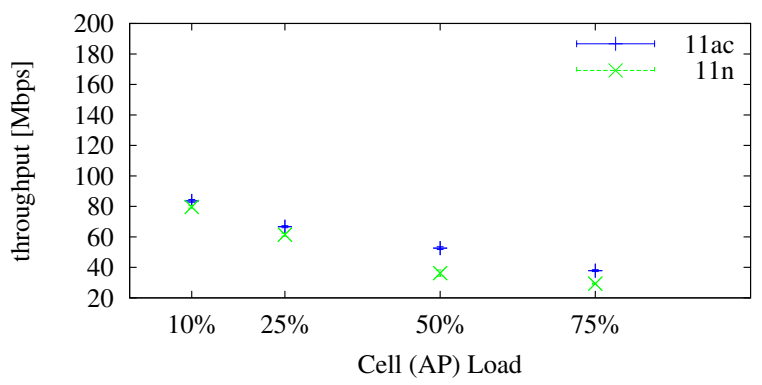

(b) Throughput

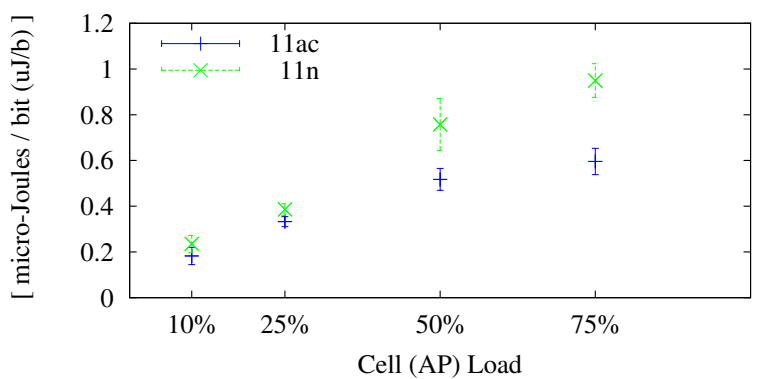

(d) Energy usage

Fig. 2. Throughput and $E_{A}$ for TCP flows for 802.11 ac and 802.11 n using $20 \mathrm{MHz}$ channel width.

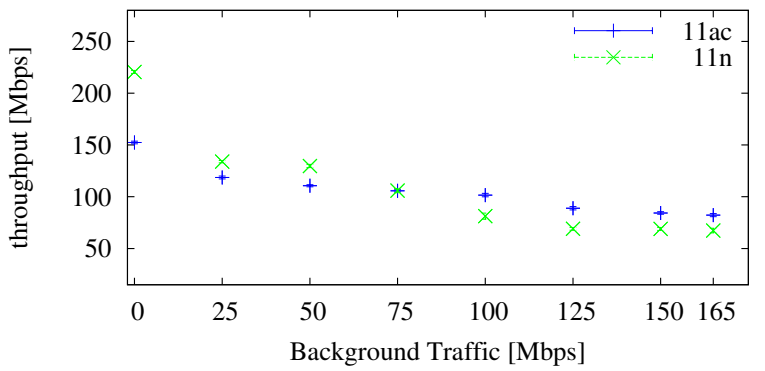

(a) Throughput

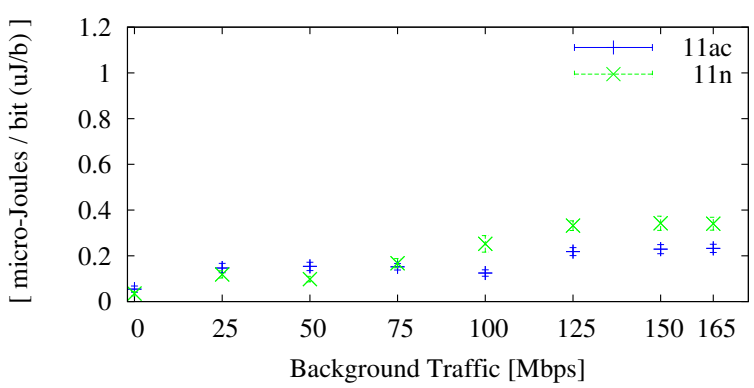

(c) Energy usage

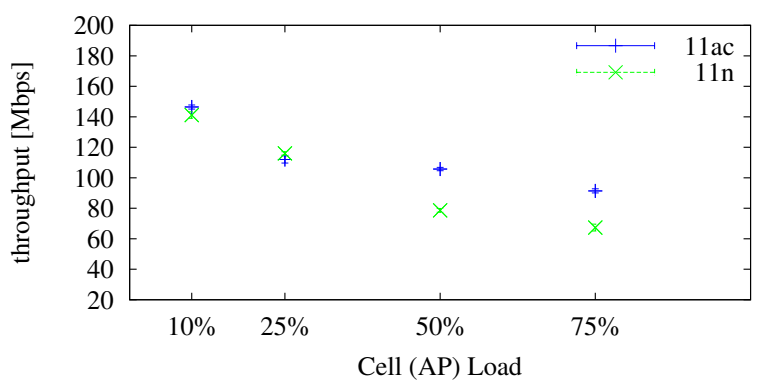

(b) Throughput

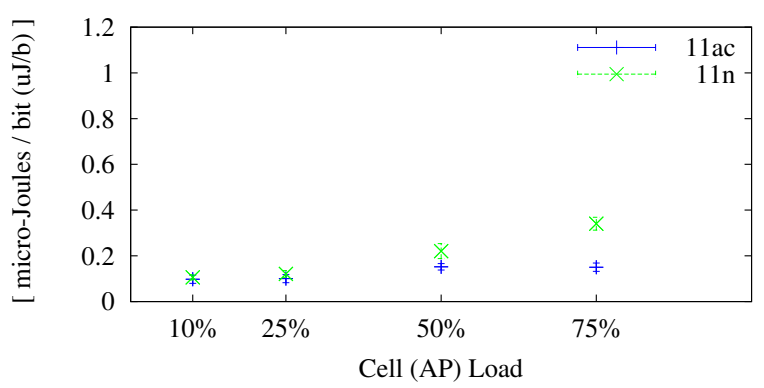

(d) Energy usage

Fig. 3. Throughput and $E_{A}$ for TCP flows 802.11 ac and 802.11 n using $40 \mathrm{MHz}$ channel width. 